\title{
MiR-139-5p/SLC7A11 Inhibits the Proliferation and Migration of Pancreatic Carcinoma via PI3K/Akt Signaling Pathway
}

Running title: MiR-139-5p regulates the cell growth and migration of pancreatic carcinoma.

Qiu-Liang Yan ${ }^{1}$, Jian-Wei Wang ${ }^{2}$, Yan Chen ${ }^{3}$, Qing-Huang $\mathrm{Ye}^{3}$, Zhi-Jiang Wang ${ }^{3}$, Jin-Hui Zhu, ${ }^{3, *}$

1 Department of General Surgery, Jinhua People's Hospital, Jinhua 321000, China

2 Department of Surgical Oncology, Second Affiliated Hospital, Zhejiang University School of Medicine, Hangzhou 310009, China

3 Department of General Surgery, Second Affiliated Hospital, Zhejiang University School of Medicine, Hangzhou 310009, China

*Corresponding Author: Jin-Hui Zhu, Department of General Surgery, Second Affiliated Hospital, Zhejiang University School of Medicine, No. 88 Jiefang Road, Shangcheng District, Hangzhou 310009, China; Tel: +86-571-87784695, Email: 2512016@zju.edu.cn 


\section{Highlights}

1. Low expression of miR-139-5p and high expression of SLC7A11 are found in both pancreatic carcinoma (PANC) tissues and cells, and the prognosis of the two above is negatively correlated.

2. Overexpression of miR-139-5p inhibits the proliferation, migration and invasion of PANC cells.

3. Overexpression of miR-139-5p regulates the proliferation, migration and invasion of PANC cells by inhibiting SLC7A11 expression.

4. MiR-139-5p affects the expression of phosphatidylinositol signaling pathway-associated proteins, PI3K and Akt, by inhibiting SLC7A11 expression.

5. Overexpression of miR-139-5p inhibits the tumor growth of PANC. 


\begin{abstract}
Objective: Pancreatic carcinoma (PANC) is one of the important aggressive cancers, with deficiency in effective therapeutics. Studies have unveiled that miR-139-5p expression is significantly downregulated in other types of cancers. However, the functions and mechanisms of miR-139-5p in PANC remain unclear.
\end{abstract}

Methods: Bioinformatic analysis was performed to analyze the differentially expressed genes in the TCGA database. PANC cell line with overexpressed miR-139-5p and Solute Carrier Family 7, Member 11 (SLC7A11) was established, and has been used to detect cell proliferation, migration, invasion and colony formation in PANC. Subsequently, bioinformatic analysis and luciferase assay were performed to confirm that SLC7A11 was a target gene of miR-139-5p. Xenograft mouse model was used to investigate the role of miR-139-5p in PANC tumorigenicity.

Results: Through bioinformatic analysis, miR-139-5p was predicted to regulate phosphatidylinositol signaling pathway by targeting SLC7A11. MiR-139-5p was found to be lowly expressed in PANC tissues, while SLC7A11 was highly expressed. Low expression of miR-139-5p and high expression of SLC7A11 were positively associated with poor clinical outcomes. PANC cell proliferation, migration, and invasion could be inhibited by miR-139-5p overexpression and could be promoted by SLC7A11 overexpression. MiR-139-5p could regulate the protein expression level of PI3K and Akt associated with phosphatidylinositol signaling pathway could be by inhibiting the expression of SLC7A11. MiR-139-5p overexpression could suppress PANC tumor growth and the expression of SLC7A11, p-PI3K, p-Akt in tumor tissues. Therefore, the inhibiting effect of miR-139-5p to PANC cell proliferation, invasion and migration, at least, was partly due to its inhibiting effect on SLC7A11 expression.

Conclusion: These results demonstrated a novel role of miR-139-5p/SLC7A11 in PANC and provided potential prognostic predictors for PANC patients.

Keywords: miR-139-5p; SLC7A11; proliferation; migration; pancreatic cancer (PANC) 


\section{Introduction}

Pancreatic cancer (PANC) is the seventh leading cause of cancer-related deaths in China, while it's the fourth cause in the United States ${ }^{1}$ with over 2,500,000 deaths every year. Because of the little progress of PANC diagnosis and therapeutic methods made in the past several decades, poor prognosis has become the leading cause to PANC as one of the most aggressive cancers ${ }^{2}$. Therefore, the development of novel methods of early diagnosis and treatments for PANC are of great important both in the study and clinic.

MicroRNA (miRNA) is a type of small RNA with about 22 nucleotides in length and widely distributed in eukaryotic cells, characterized with highly conservativity in evolution progress and regulatory functions in cell differentiation, proliferation and apoptosis $^{3,4}$. Studies have shown that miRNA expression is abnormal in tissues of various human tumors, which is significantly different in normal tissues, with distinct tissue specificity that could be used to determine the origin of tumors ${ }^{5}$. Therefore, miRNA plays an important role in differentiation and migration of tumor. In the study of tumor miRNA, miR-139-5p has been widely regarded as an important member of miRNA family,7. MiR-139-5p is located in the second intron of the phosphodiesterase $2 \mathrm{~A}$ gene on chromosome $11 \mathrm{q} 13.4$ with $23 \mathrm{nt}$ in $\operatorname{size}^{6,8}$.It is a common type of mature miRNA generated from a miR-139 precursor ,and has been found to be lowly expressed in various cancers, such as malignant glioblastoma ${ }^{9}$, bladder cancer ${ }^{10}$, hepatic carcinoma ${ }^{11}$ and breast cancer ${ }^{12}$. Moreover, overexpression of miR-139-5p could inhibit cancer cell proliferation, invasion and migration, but the functions and mechanisms of miR-139-5p in PANC cells are little studied.

MiRNA generally regulates tumor growth and migration by regulating target genes. Solute Carrier Family 7, Member 11 (SLC7A11), a subunit of amino acid transport system $\mathrm{X}_{\mathrm{c}}^{-}$, mainly functions in regulating cystine into cells during glutamate exchange ${ }^{13,14}$. The raw material of glutathione is synthesized in the intracellular of cysteine, and the reduce of glutathione can regulate cycytoxicity ${ }^{15,16}$. Previous studies have shown that SLC7A11 is related to cancer lesions and 
occurrence, and its expression has been found to be correlated with poor prognosis in various malignancies. Okuno, et al. found that $\mathrm{X}_{\mathrm{c}}^{-}$system could strengthen the resistance of ovarian cancer on platinum compound ${ }^{15}$. Huang, et al. found that SLC7A11 expression can be used as a predictor of cells on the drug resistance of geldanamycin mediated by L-alanosine and glutathione, which provides potential targets for improving the chemosensitivity of various drugs ${ }^{17}$. Therefore, SLC7A11 plays an important role in cancer cell proliferation and migration.

In the present study, we investigated the target relationship between miR-139-5p and SLC7A11. Then we studied the regulatory effect of miR-139-5p/SLC7A11 on the expression of PI3K/Akt. We also identify the role of miR-139-5p/SLC7A11 in the proliferation and migration of PANC cell line. These results can help researchers develop novel therapeutic approaches.

\section{Material and Methods}

\section{Bioinformatic Analysis}

The miRNA and mRNA expression data of TCGA-PAAD were downloaded from the TCGA database, and the difference analysis $(|\operatorname{logFC}|>2, \operatorname{padj}<0.05)$ was performed by edgeR. The clinical information of the sample was used to analyze the survival of the differential miRNA to determine the target miRNA of the study. The target miRNA was targeted predicted using the starBase database, and the differential mRNA was taken intersection to obtain the target gene. Pathological enrichment analysis of target miRNAs and their target genes was performed using GSEA software.

\section{Clinical data and organizational sources}

We collected 45 pancreatic cancer tissues and adjacent nontumour tissues tissues that were acquired from July 2018 to July 2019. All registered patients met the following criteria: (a) clear pathological diagnosis of pancreatic cancer, (b) Have signed a pre-operative informed letter. The clinical and pathological data of patients are shown in Schedule 1.

\section{Cell and cell culture}


The cell line information used in this study is shown in Table 2. HPDE, PANC-1, Bx-PC3 cells were cultured in DMEM complete medium with 10\% FBS (Gibco, Grand Island, NY, USA) and $100 \mathrm{U} / \mathrm{ml}$ penicillin/streptomycin (Corning, New York, NY, USA) (sigma, USA) and was cultured at $37^{\circ} \mathrm{C}$ in a $5 \% \mathrm{CO}_{2}$ incubator.

\section{Cell transfection}

100 nmol/L miR-139-5p mimic (miR-139-5p), 100 nmol/L oe-SLC7A11 (SLC7A11) and their negative controls (NC-mimic and oe-NC) were purchased from GenePharma (Shanghai), China). Approximately $1 \times 10^{5}$ cells were seeded into 12-well plates. Oe-SLC7A11, miR-139-5p mimic and negative controls were transfected into cells using the LipoFiter kit (Hanbio, Shanghai, China) according to the manufacturer's protocol. RNA and protein were extracted $48 \mathrm{~h}$ after transfection. The synthesized primer sequences are shown in Table 3.

\section{Real-time PCR（qRT-PCR）}

Total RNA was extracted from frozen tissue using Trizol (Invitrogen) according to the manufacturer's protocol. cDNA was synthesized using a reverse transcription system kit (Invitrogen). qRT-PCR was performed on an ABI 7900HT instrument (Applied Biosystems, USA). Quantitative PCR was performed using the miScript SYBR Green PCR Kit (Qiagen, Germany) under the following thermal cycler conditions: $95^{\circ} \mathrm{C}$ for $2 \mathrm{~min}, 95^{\circ} \mathrm{C}$ for $5 \mathrm{~s}$ and $60^{\circ} \mathrm{C}$ for $30 \mathrm{~s}$ for 40 cycles. SLC7A11 and miR-139a-5p was normalized using GAPDH and U6 as internal reference respectively. The primers used are shown in Table 3. The $2^{-\Delta \Delta \mathrm{Ct}}$ value was used to compare the difference in mRNA expression of the target gene between the control group and the experimental group. The experiment was repeated 3 times.

\section{Western blot assay}

After transfection for $48 \mathrm{~h}$ in different treatment groups, each group of cells was washed 3 times with cold PBS (Thermo Fisher, USA), added with whole protein lysate, and lysed on ice for $10 \mathrm{~min}$. Protein quantification was performed using BCA Quantitation Kit (Thermo fisher, USA). $10 \mu$ of the upper buffer was added at $95{ }^{\circ} \mathrm{C}$ for $10 \mathrm{~min}$, and then subjected to SDS-PAGE at $100 \mathrm{~V}$. After electrophoresis, the protein was transferred to the $\mathrm{NC}$ membrane at $100 \mathrm{~mA}$ for $120 \mathrm{~min}$, blocked with 
blocking solution (5\% BSA/TBST) for $60 \mathrm{~min}$, and primary antibody was added and incubated at $4{ }^{\circ} \mathrm{C}$ overnight. After incubation of the primary antibody, the membrane was washed with a $1 \times$ TBST solution (Solarbio, Beijing, China) at room temperature under a shaking table for $5 \min \times 3$ times, horseradish peroxidase-labeled secondary anti-goat anti-rabbit IgG was added and hybridized at room temperature. After 120 min, TBST was washed 3 times. After $20 \mathrm{~min}$, the luminescence reaction was carried out with an ECL kit (Solarbio, Beijing, China) and the protein imprint was photographed. The experiment was repeated three times. The antibody information used in the experiment is shown in Table 4.

\section{Cell proliferation, EdUand colony formation assay}

Cell proliferation was tested by MTT assay. PANC-1, Bx-PC3 cells ( 5 x $10^{3}$ per $100 \mathrm{ul}$ ) were seeded into 96-well plates. Each treatment included 3 repetitions. After 1, 2, 3, 4 and 5 days of culture, the cells were evaluated for cell proliferation using a sterile MTT solution (Beyptime) according to the method described in description. A spectrophotometer (Molecular Devices, Sunnyvale, CA, USA) were used to measure the absorbance at $450 \mathrm{~nm}$. The cell DNA synthesis rate was measured using the EdU kit (Ribobio, Guangzhou, China) according to the manufacturer's protocol. EdU-positive cells were observed using a microscope, and 3 fields of view was randomly selected in each well. The ratio of EdU positive cells (red fluorescence) to Hoechest stained cells (blue fluorescence) was used to evaluate cell proliferation activity. In the colony formation experiment, each treatment was 6 wells, 1000 cells per well. After 14 days of culture, 30\% formaldehyde (Thermo fisher, USA) was added to the cells. After 15 minutes, $0.1 \%$ crystal violet (Thermo fisher, USA) was used for dyeing. The number of clones was calculated using an optical microscope (more than 50 cells per clone).

\section{Wound healing assay and Transwell invasion assay}

In the wound healing assay, PANC-1, Bx-PC3 cells $\left(5 \times 10^{6}\right)$ were seeded on 6-well plates. When the cell coverage reached $80 \%$, a 200 ul pipette tip was used to gently scrape through the single layer in the center of the well. The wells were briefly washed twice with medium to remove the isolated cells. Added with fresh medium the 
cells were regenerated for $48 \mathrm{~h}$. The cell migration was observed and photographed on the microscope for $0 \mathrm{~h}$ and $48 \mathrm{~h}$. In cell invasion assay, a 24-well Transwell chamber (8 $\mu \mathrm{m}$ pore size, BD Biosciences) was used for the transwell invasion assay. Approximately $2 \times 10^{4}$ cells were added to the upper chamber and the top chamber was coated with Matrigel matrix. Dulbecco's Modified Eagle Medium (DMEM) containing 10\% fetal bovine serum (FBS) (Thermo fisher, USA) was filled into the lower chamber. After incubation at $37^{\circ} \mathrm{C}$ for $48 \mathrm{~h}$, the cells that failed the membrane were removed with a cotton swab applicator and the cells on the lower surface of the membrane were stained with $0.5 \%$ crystal violet.

\section{Dual luciferase reporter assay}

The psiCHECK luciferase reporter vector (Sangon Co., LTD, ShangHai, China) inserted with SLC7A11 wild type (wt) and mutant (mut) 3'UTR was constructed. HEK293T cells (Thermo fisher, USA) were seeded in 48-well plates and cultured for $24 \mathrm{~h}$. The miR-139-5p/NC and psiCHECK wt/mut plasmids were co-transfected into the cells. Finally, luciferase activity was measured by a luciferase assay reagent (Promega, Fitchburg, WI, USA).

\section{Tumor xenograft experiments}

Twelve male nude mice (4 weeks) were purchased from Heavy River Experimental Technology Co., Ltd. (Beijing, China) and kept under sterile conditions ( $12 \mathrm{~h}$ black and white photoperiod, $25^{\circ} \mathrm{C}, 60 \%-70 \%$ humidity). The human source miR-139-5p sequence was amplified and ligated into pcDNA3.1(+) to form a miR-139-5p expression vector (GenePharma, ShangHai, China). A blank pcDNA3.1 vector was served as a control. Lentiviral-encapsulated miR-139-5p or blank lentivirus was transfected into PANC-1 cells for $96 \mathrm{~h}$, then treated with puromycin for 4 weeks for cell selection (Santa Cruz Bio). The transfected miR-139-5p and NC-mimic groups were injected subcutaneously into each group of nude mice. The test was started 1 week after tumor growth. Tumor volume measurement method: volume $(\mathrm{cm} 3)=(\text { length } \mathrm{x} \text { width })^{2} / 2$. After 4 weeks, the nude mice were euthanized. The tumor weight was measured and photographed.

\section{Statistical analysis}


All data were processed by SPSS 22.0 statistical software. The measurement data were presented as mean \pm standard deviation. The comparison between the two groups was $t$ test. The comparison between multiple groups should be analyzed by one-way ANOVA. $P<0.05$ indicates that the difference is statistically significant, and $P<0.01$ indicates an extremely significant difference.

\section{Results}

\section{Bioinformatics predicted miR-139-5p regulate SLC7A11 related pathways}

Six differentially expressed miRNAs and 301 differentially expressed mRNAs were obtained by edgeR differential analysis (Figure 1A). Survival analysis of differential miRNA showed that the low expression of miR-139 in tumor tissues had a significant impact on prognosis, and the survival time of patients with low expression of miR-139 was significantly lower than that of patients with high expression of miR-139 (Figure 1B). Targeting genes of miR-139-5p, which has been identified mature sites in miR-139 and is most important for prognosis, were predicted by starBase database. After intersection with 26 up-regulated differentially expressed mRNAs, 4 differentially expressed mRNAs (SLC7A11, B3GNT3, SH3TC2, CHRNA5) with binding sites of miR-139-5p were obtained (Figure 1C). SLC7A11, which has a significant effect on prognosis and plays an important role in cancer prognosis, was selected for in-depth analysis as the targeting gene of miR-139-5p in this study (Figure 1D). The GSEA pathway enrichment analysis of miR-139 and its target genes found that miR-139 was mainly concentrated in the phosphatidylinositol signaling system, calcium signaling pathway and cell adhesion molecule CAMS signaling pathway (Attached map 1A). Excessive activation of phosphatidylinositol will lead to abnormal cell proliferation, metastasis, endocytosis and exocytosis, and even tumor occurrence. However, SLC7A11 mainly concentrated on pathways such as endocytosis and tight junctions (Attached map 1B). Therefore, miR-139-5p is likely to regulate the phosphatidylinositol signaling system to inhibit tumor proliferation and metastasis by down-regulating SLC7A11. 


\section{In clinical tissues and PANC cells of PANC patients, the expression of miR-139-5p is low while expression of SLC7A11 is high}

From the survival curve, it was found that miR-139-5p expression was positively correlated with patient survival, while SLC7A11 was negatively correlated. We studied from tissue and cell levels to further explore the relationship between the expression of miR-139-5p and SLC7A11 and PANC. The expression of miR-139-5p and SLC7A11 in PANC tissue samples and adjacent tissues as well as the protein expression of SLC7A11 were detected at the tissue level. The results showed that the expression level of miR-139-5p in PANC tissues was lower than that in adjacent tissues (Figure 2A), and the mRNA and protein expression levels of SLC7A11 in PANC tissues were higher than that in adjacent tissues (Figure 2B, C). To study the relationship between the expression of miR-139-5p and clinicopathology, the relative expression level of miR-139-5p in pancreatic cancer tissues was ranked from high to low, and the median was taken as the cutoff point to divide 45 patients into two groups: high and low expression. The results showed that there was no significant correlation between miR-139-5p and clinical parameters such as gender, age, tumor size, tumor site, differentiation degree, TNM staging and lymphatic metastasis $(\mathrm{P}>0.05)$ (Table 1). The correlation analysis of the expression of miR-139-5p and SLC7A11 tissues showed that the expression of miR-139-5p and SLC7A11 was significantly negatively correlated $(\mathrm{P}<0.01)$ (Figure 2D). The expression of miR-139-5p and SLC7A11 in human pancreatic duct epithelial cells HPDE and two pancreatic cancer cells PANC-1 and Bx-PC3 were compared at the cellular level. The results showed that the expression of miR-139-5p in the two kinds of PANC cells was significantly lower than that in HPDE (Figure 2E), while the mRNA and protein expression of SLC7A11 in the two kinds of PANC cells was significantly higher than that in HPDE (Figure 2F, G).

Overexpression of miR-139-5p inhibited proliferation, migration and invasion of PANC cells

The expression of miR-139-5p in PANC clinical tissues and cells shows that there is a negative correlation. And we infer that overexpression of miR-139-5p in 
PANC cells will inhibit cell proliferation, migration and invasion. To verify the possibility, we examined the effects of overexpression of miR-139-5p on proliferation, migration and invasion of PANC-1 and Bx-PC3 cancer cells. MTT results showed that the overexpression of miR-139-5p significantly reduced the viability of both types of cells (Figure 3A, B). Cloning formation experiments also showed that overexpression of miR-139-5p significantly inhibited the growth ability of PANC cells (Figure 3C, D). In addition, the EdU assay showed that miR-139-5p overexpression reduced the reproductive activity of cancer cells (Figure 3E, F). We further measured the effect of miR-139-5p on PANC cell migration and invasion, and found that the migration and invasion ability of PANC cells were significantly inhibited after the overexpression of miR-139-5p (Figure 3G-J). These results indicated that miR-139-5p as a tumor suppressor could inhibit the proliferation, migration and invasion of PANC cells.

\section{MiR-139-5p regulates PANC cell proliferation, migration and invasion by targeting SLC7A11}

After confirming the anti-cancer function of miR-139-5p, we further studied the potential target genes of miR-139-5p. Online miRNA data analysis software (starBase) showed that SLC7A11 was a direct target gene of miR-139-5p according to the 3 'UTR predicted target binding site of SLC7A11 (Figure 4A). Results of Western blot and qRT-PCR showed that overexpression of miR-139-5p inhibited the expression of SLC7A11 (Figure 4B, C). In addition, luciferase assay results also showed that overexpression of miR-139-5p inhibited the luciferase activity of wild-type SLC7A113 'UTR without affecting the luciferase activity of mutated SLC7A113' UTR (Figure 4D). These results showed that miR-139-5p directly targets SLC7A11 and inhibits the expression of SLC7A11.

In the study of the effect of SLC7A11 targeted by miR-139-5p on PANC cell proliferation, migration and invasion, the MTT results showed that the overexpression of SLC7A11 significantly increased the survival activity of the two cancer cells $(\mathrm{P}<0.05)$, but when SLC7A11 and miR-139-5p were overexpressed simultaneously, the survival activity of the two cancer cells was significantly decreased (Figure 5A, B). The results of cell cloning formation experiment also showed that overexpression 
of SLC7A11 significantly increased the number of clone formation of the two cancer cells $(\mathrm{P}<0.05)$, but when SLC7A11 and miR-139-5p were overexpressed simultaneously, the number of clone formation of the two cancer cells was significantly decreased (Figure 5C, D). We further measured the migration and invasion of the two PANC cancer cells and found that the migration and invasion of PANC-1 and Bx-PC3 cells significantly increased after SLC7A11 overexpression $(\mathrm{P}<0.05)$, but the migration and invasion of the two cancer cells was significantly inhibited when SLC7A11 and miR-139-5p were overexpressed simultaneously (Figure 5E- H). These results suggest that miR-139-5p regulates cell proliferation, migration, and invasion by inhibiting SLC7A11 expression.

\section{The miR-139-5p/SLC7A11 axis regulated the PI3K/Akt signaling pathway}

Analysis of the GSEA pathway found that miR-139-5p was concentrated in the pathways of phosphatidylinositol signaling related functions (Attached map 1), and SLC7A11 mainly concentrated in the pathways of endocytosis and tight junctions, which have an impact on the proliferation of cancer cells and tumors when phosphatidylinositol is over-activated. Previous studies on many other tumors have also found that miR-139-5p regulates phosphatidylinositol 3-kinase (PI3K) and its downstream gene protein kinase B (Akt), which is closely related to phosphatidylinositol signal ${ }^{8,18}$. In order to explore whether miR-139-5p in pancreatic cancer cells could regulate the PI3K/Akt signaling pathway by targeting SLC7A11, we overexpressed and co-overexpressed miR-139-5p and SLC7A11 in PANC-1 and Bx-PC3, respectively, to observe the effects on PI3K and Akt. The results showed that the overexpression of miR-139-5p in PANC-1 and Bx-PC3 cells significantly reduced the expression levels of p-PI3K and p-Akt, while the overexpression of SLC7A11 significantly increased the expression levels of p-PI3K and p-Akt. When miR-139-5p and SLC7A11 were overexpressed simultaneously, the expression levels of p-PI3K and p-Akt were not significantly different from the blank group (Figure 6A and B). This result suggests that miR-139-5p may regulate PI3K/Akt targeting by inhibiting SLC7A11.

MiR-139-5p overexpression suppressed PANC tumour proliferation in vivo 
In order to study the effect of miR-139-5p on the growth of PANC cells in vivo, we selected PANC-1 cells as in vivo injection cells to construct lentivirus-coated overexpressed miR-139-5p cell lines and injected them into the subcutaneous of mice. The blank control group was the blank lentivirus PANC-1 cells. The results showed that tumor growth was significantly inhibited in the miR-139-5p overexpression treatment group compared with the blank group $(\mathrm{P}<0.05)$ (Figure 7B). Compared with the blank group, the tumor volume and weight of the miR-139-5p overexpression group were significantly smaller in nude mice (Figure 7A, C). These results showed that miR-139-5p had a significant inhibitory effect on the growth of PANC tumors. However, SLC7A11, p-PI3K and p-Akt signal related proteins were significantly inhibited in tumor tissues of nude mice after nc-mimic or overexpression of miR-139-5p (Figure 7D), which was consistent with the results in PANC cells.

\section{Discussion}

MiRNA involves in multiple tumor progresses, and it's important to know the biological functions on miRNA regulating oncogenes and antioncogenes ${ }^{19,20}$. The regulatory effect of miR-139-5p on tumor growth has be confirmed in various cancers $^{8,21,22}$, but the biological functions and molecular mechanisms of miR-139-5p in PANC has not been focused. In our study, we confirmed that miR-139-5p was lowly expressed in both PANC tissues and cells. Through functional analysis, overexpression of miR-139-5p significantly inhibited PANC cell proliferation, invasion and migration, and the inhibition of miR-139-5p overexpression in tumor growth of nude mouse PANC was found in vivo experiment. Results above revealed that miR-139-5p acts as an antioncogene in PANC, which is consistent with the results in other types of cancers. Shen, et al. found that miR-139 could inhibit the invasion and migration of colorectal cancer cells by targeting I type insulin-like growth factor ${ }^{23}$. Zhang, et al. found that miR-139-5p could inhibit the development of colorectal cancer by targeting $\mathrm{NOTCH} 1^{24}$. And Gu, et al. found that miR-139 could inhibit the development of hepatic carcinoma by regulating WNT-TCF-4 pathway ${ }^{25}$. 
In view of the findings, miR-139-5p plays an important role in cell growth and migration of PANC acting as a tumor suppressor.

Through bioinformatic analysis and luciferase assay, miR-139-5p is confirmed to targeted regulate SLC7A11. In addition, considering the expression of miR-139-5p and SLC7A11 in tissues and cells, their expressions were negatively correlated in PANC. Transport system $\mathrm{X}_{\mathrm{c}}^{-}$, combination of SLC7A11 and SLC3A2, plays an important role in cell growth and drug resistance of PANC ${ }^{13,26}$. In this study, we found that overexpression of SLC7A11 could significantly promote PANC cell proliferation and migration, which is consistent with the previous studies in other cancers ${ }^{5}$. Moreover, when miR-139-5p and SLC7A11 were overexpressed simultaneously, the promotion effect of SLC7A11 on PANC cell proliferation and migration was significantly inhibited. Results above indicated that the regulatory effect of miR-139-5p on PANC cells is partly processed by targeting SLC7A11.

$\mathrm{PI} 3 \mathrm{~K} / \mathrm{Akt}$, one of the important signal transduction pathways in cells, regulates cell growth by affecting the activation state of multiple effectors in the downstream, and plays an important role in inhibiting cell apoptosis and promoting proliferation ${ }^{27,28}$. The regulatory effect of miR-139-5p on PI3K/Akt signaling pathway has been reported in other cancers. For example, Catanzaro, et al. found that miR-139-5p regulates the proliferation of glioma cells by targeting PI3K/AKT/mTORC1 signaling pathway ${ }^{18}$. Krishman, et al. found that miR-139-5p involves in various pathways, including PI3K/Akt, in breast cancer ${ }^{8}$. In our study, the enrichment results of GSEA pathway for miR-139-5p and SLC7A11 revealed that both miR-139-5p and SLC7A11 enriched in PI3K/Akt-associated signaling pathways. In addition, the protein expression of PI3K/Akt after overexpressing miR-139-5p and SLC7A11 showed that miR-139-5p negatively regulated PI3K/Akt in PANC, which was partly due to the targeting action on SLC7A11.

In conclusion, this study confirmed the negatively regulatory effect of miR-139-5p on PANC cells. Through targeting SLC7A11, miR-139-5p significantly inhibited PANC cell proliferation, invasion and migration, and regulated PI3K/Akt 
signaling pathway. The results can help people to learn more about the role of miR-139-5p in PANC, and to find novel targeted therapies. 


\section{Statements}

\section{Acknowledgement}

None.

\section{Statement of Ethics}

The authors have no ethical conflicts to disclose.

\section{Conflict of interest statement}

The authors have no conflicts of interest to declare.

\section{Funding Sources}

This study was supported in part by Funds of Science Technology Department of Zhejiang Province (2016C37103), National Science Foundation of China (81201733), Zhejiang Province Bureau of Health (2015121608), Science Technology Department of Jinhua City (2015-3-013),

\section{Author Contributions}

Dr Zhu JH, Yan QL and Wang JW contributed to the study design. Chen Y conducted the literature search. Ye QH and Wang ZJ acquired the data. Zhu JH and Yan QL wrote the article. Yan QL, Chen Y and Wang JW performed data analysis. Zhu JH and Yan QL revised the article and gave the final approval of the version to be submitted. All authors read and approved the final manuscript.

\section{Availability of data and material}

The data used to support the findings of this study are available from the corresponding author upon request. 


\section{Consent for publication}

Not applicable 


\section{Reference}

1 Zhang, M. L. et al. [Report of breast cancer incidence and mortality in China registry regions, 2008-2012]. Zhonghua Zhong Liu Za Zhi 41, 315-320, doi:10.3760/cma.j.issn.0253-3766.2019.04.013 (2019).

2 Gillen, S., Schuster, T., Meyer Zum Buschenfelde, C., Friess, H. \& Kleeff, J. Preoperative/neoadjuvant therapy in pancreatic cancer: a systematic review and meta-analysis of response and resection percentages. PLoS Med 7, e1000267, doi:10.1371/journal.pmed.1000267 (2010).

3 Zheng, H., Liu, J. Y., Song, F. J. \& Chen, K. X. Advances in circulating microRNAs as diagnostic and prognostic markers for ovarian cancer. Cancer Biol Med 10, 123-130, doi:10.7497/j.issn.2095-3941.2013.03.001 (2013).

4 Summerer, I. et al. Changes in circulating microRNAs after radiochemotherapy in head and neck cancer patients. Radiat Oncol 8, 296, doi:10.1186/1748-717X-8-296 (2013).

5 Wu, Y., Sun, X., Song, B., Qiu, X. \& Zhao, J. MiR-375/SLC7A11 axis regulates oral squamous cell carcinoma proliferation and invasion. Cancer Med 6, 1686-1697, doi:10.1002/cam4.1110 (2017).

6 Chen, J. et al. MiR-139-5p is associated with poor prognosis and regulates glycolysis by repressing PKM2 in gallbladder carcinoma. Cell Prolif 51, e12510, doi:10.1111/cpr.12510 (2018).

7 Huang, L. L. et al. Potential role of miR-139-5p in cancer diagnosis, prognosis and therapy. Oncol Lett 14, 1215-1222, doi:10.3892/ol.2017.6351 (2017).

8 Krishnan, K. et al. miR-139-5p is a regulator of metastatic pathways in breast cancer. RNA 19, 1767-1780, doi:10.1261/rna.042143.113 (2013).

9 Bushati, N. \& Cohen, S. M. microRNA functions. Annu Rev Cell Dev Biol 23, 175-205, doi:10.1146/annurev.cellbio.23.090506.123406 (2007).

10 Adam, L. et al. miR-200 expression regulates epithelial-to-mesenchymal transition in bladder cancer cells and reverses resistance to epidermal growth factor receptor therapy. Clin Cancer Res 15, 5060-5072, doi:10.1158/1078-0432.CCR-08-2245 (2009).

11 Qiu, G., Lin, Y., Zhang, H. \& Wu, D. miR-139-5p inhibits epithelial-mesenchymal transition, migration and invasion of hepatocellular carcinoma cells by targeting ZEB1 and ZEB2. Biochem Biophys Res Commun 463, 315-321, doi:10.1016/j.bbrc.2015.05.062 (2015).

12 Asangani, I. A. et al. MicroRNA-21 (miR-21) post-transcriptionally downregulates tumor suppressor Pdcd4 and stimulates invasion, intravasation and metastasis in colorectal cancer. Oncogene 27, 2128-2136, doi:10.1038/sj.onc.1210856 (2008).

13 Sato, H., Tamba, M., Ishii, T. \& Bannai, S. Cloning and expression of a plasma membrane cystine/glutamate exchange transporter composed of two distinct proteins. $J$ Biol Chem 274, 11455-11458, doi:10.1074/jbc.274.17.11455 (1999). 
14 Bannai, S. Transport of cystine and cysteine in mammalian cells. Biochim Biophys Acta 779, 289-306, doi:10.1016/0304-4157(84)90014-5 (1984).

15 Okuno, S. et al. Role of cystine transport in intracellular glutathione level and cisplatin resistance in human ovarian cancer cell lines. $\mathrm{Br} J$ Cancer 88, 951-956, doi:10.1038/sj.bjc.6600786 (2003).

16 Lu, S. C. Glutathione synthesis. Biochim Biophys Acta 1830, 3143-3153, doi:10.1016/j.bbagen.2012.09.008 (2013).

17 Huang, Y., Dai, Z., Barbacioru, C. \& Sadee, W. Cystine-glutamate transporter SLC7A11 in cancer chemosensitivity and chemoresistance. Cancer Res 65, 7446-7454, doi:10.1158/0008-5472.CAN-04-4267 (2005).

18 Catanzaro, G. et al. The miR-139-5p regulates proliferation of supratentorial paediatric low-grade gliomas by targeting the PI3K/AKT/mTORC1 signalling. Neuropathol Appl Neurobiol 44, 687-706, doi:10.1111/nan.12479 (2018).

19 Chi, Y. et al. CDK11p58 inhibits ERalpha-positive breast cancer invasion by targeting integrin beta 3 via the repression of ERalpha signaling. BMC Cancer 14, 577, doi:10.1186/1471-2407-14-577 (2014).

20 Chi, Y. et al. Abnormal expression of CDK11p58 in prostate cancer. Cancer Cell Int 14, 2, doi:10.1186/1475-2867-14-2 (2014).

21 Zhang, Y. et al. Involvement of aberrant miR-139/Jun feedback loop in human gastric cancer. Biochim Biophys Acta 1853, 481-488, doi:10.1016/j.bbamcr.2014.12.002 (2015).

22 Luo, H. N. et al. MiR-139 targets CXCR4 and inhibits the proliferation and metastasis of laryngeal squamous carcinoma cells. Med Oncol 31, 789, doi:10.1007/s12032-013-0789-z (2014).

23 Shen, K. et al. MiR-139 inhibits invasion and metastasis of colorectal cancer by targeting the type I insulin-like growth factor receptor. Biochem Pharmacol 84, 320-330, doi:10.1016/j.bcp.2012.04.017 (2012).

24 Zhang, L. et al. microRNA-139-5p exerts tumor suppressor function by targeting NOTCH1 in colorectal cancer. Mol Cancer 13, 124, doi:10.1186/1476-4598-13-124 (2014).

$25 \mathrm{Gu}$, W., Li, X. \& Wang, J. miR-139 regulates the proliferation and invasion of hepatocellular carcinoma through the WNT/TCF-4 pathway. Oncol Rep 31, 397-404, doi:10.3892/or.2013.2831 (2014).

26 Lo, M., Ling, V., Wang, Y. Z. \& Gout, P. W. The xc- cystine/glutamate antiporter: a mediator of pancreatic cancer growth with a role in drug resistance. Br J Cancer 99, 464-472, doi:10.1038/sj.bjc.6604485 (2008).

27 Burris, H. A., 3rd. Overcoming acquired resistance to anticancer therapy: focus on the PI3K/AKT/mTOR pathway. Cancer Chemother Pharmacol 71, 829-842, doi:10.1007/s00280-012-2043-3 (2013).

28 Hassan, B., Akcakanat, A., Holder, A. M. \& Meric-Bernstam, F. Targeting the PI3-kinase/Akt/mTOR signaling pathway. Surg Oncol Clin N Am 22, 641-664, doi:10.1016/j.soc.2013.06.008 (2013). 


\section{Figure legends}

Figure 1 Expression prognosis and targeting relationship of miR-139-5p and SLC7A11

A: TCGA database differential gene heat map of normal and tumor groups in pancreatic cancer, with differential miRNAs at the top and differential mRNAs at the bottom; B: The survival curve of the expression level of miR-139 on the prognosis of patients, the red line is the group with high expression, and the blue line is the group with low expression; C: Venny plots of miR-139-5p predicted target genes and differential mRNAs; D: For SLC7A11 survival curve, the red line represents the group with high expression, while the blue line represents the group with low expression.

Figure 2 MiR-139-5p has low expression in clinical tissues and PANC cells of PANC patients while SLC7A11 has high expression

A: The expression of miR-139-5p in PANC tissues and paracancer tissues was detected by qRT-PCR; B: MRNA expression of SLC7A11 in PANC tissues and paracancer tissues was detected by qRT-PCR; C: The protein expression of SLC7A11 in PANC tissues and paracancer tissues was detected by western blot; D: Expression relationship between miR-139-5p and SLC7A11 tissues; E: The expression of miR-139-5p in HPDE, PANC-1 and Bx-PC3 cells was detected by qRT-PCR; F: The mRNA expression of SLC7A11 in HPDE, PANC-1 and Bx-PC3 cells was detected by qRT-PCR; G: The protein expression of SLC7A11 in HPDE, PANC-1 and Bx-PC3 cells was detected by western blot.

Note: “*” represents $\mathrm{P}<0.05$, “**” represents $\mathrm{P}<0.01$.

Figure 3 Overexpression of miR-139-5p inhibited proliferation, migration and invasion of PANC cells

A, B: The growth activity of PANC-1 and Bx-PC3 cells after miR-139-5p expression was measured by MTT assay; C, D: The growth of PANC-1 and Bx-PC3 cells after the overexpression of miR-139-5p was tested by cloning formation test; E, F: PANC-1 and Bx-PC3 cells proliferated after miR-139-5p was overexpressed by EdU assay; G, 
$\mathrm{H}$ : Invasion ability of PANC-1 and Bx-PC3 cells after overexpression of miR-139-5p was detected by transwell assay $(200 \times)$; I, J: The migration ability of PANC-1 and Bx-PC3 cells after the overexpression of miR-139-5p was detected by scratch healing experiment.

Note: “*” represents $\mathrm{P}<0.05$, “**” represents $\mathrm{P}<0.01$.

\section{Figure 4 MiR-139-5p targets SLC7A11}

A: Bioinformatics analysis proved that miR-139-5p targeted SLC7A11 binding site at 3 'UTR; B, C: SLC7A11 expression in PANC-1 and Bx-PC3 cells after the overexpression of miR-139-5p was detected by qRT-PCR and western blot; D: The overexpression of miR-139-5p affected luciferase activity in different treatment groups.

Note: “**” represents $\mathrm{P}<0.01$.

Figure 5 Effects of miR-139-5p /SLC7A11 signaling axis on PANC cell proliferation, migration and invasion

A, B: The cell activity of PANC-1 and Bx-PC3 was evaluated by MTT method; C, D: The cell growth of PANC-1 and Bx-PC3 was evaluated by cell cloning experiments; E, F: Transwell assay was used to evaluate the invasion ability of PANC-1 and Bx-PC3 cells in each treatment group; G, H: Evaluation of PANC-1 and Bx-PC3 cell migration ability after treatment by scratch healing experiment $(200 \times)$.

Note: “*” represents $\mathrm{P}<0.05$, “**” represents $\mathrm{P}<0.01$.

Figure 6 MiR-139-5p targets SLC7A11 and regulates the expression of PI3K and Akt in PANC cells

A, B: Phosphorylated PI3K (p-PI3K), total PI3K (PI3K), phosphorylated Akt (p-Akt) and total Akt (Akt) protein expression in PANC-1 and Bx-PC3 cells.

Figure 7 Overexpression of miR-139-5p inhibited PANC tumor growth in nude mice

A: MiR-139-5p inhibited PANC tumor growth in nude mice; B: The tumor growth curve of PANC in nude mice after overexpression of miR-139-5p and blank treatment was mean \pm standard deviation ( $n=6 /$ group); $C$ : Tumor weight in the blank group and the overexpressed miR-139-5p group; D: SLC7A11 and PI3K/Akt signaling pathway 
related proteins were expressed in tumor tissues of nude mice after overexpression of NC-mimic and miR-139-5p.

Note: “*” represents $\mathrm{P}<0.05$, “**” represents $\mathrm{P}<0.01$.

\section{Attached map 1 Enrichment pathways of miR-139 and SLC7A11}

A: GSEA pathway enrichment analysis results of miR-139; B: GSEA pathway enrichment analysis results of SLC7A11.

Table 1 Correlation analysis between the expression level of miR-139-5p in pancreatic cancer tissues and pathological parameters

\begin{tabular}{|c|c|c|c|c|c|}
\hline \multirow[b]{2}{*}{ Clinicopathologic factors } & \multirow[b]{2}{*}{$\begin{array}{c}\text { Number of } \\
\text { cases }\end{array}$} & \multicolumn{2}{|c|}{ miR-139-5p } & \multirow[b]{2}{*}{$\chi^{2}$ value } & \multirow[b]{2}{*}{$\begin{array}{c}\mathrm{P} \\
\text { value }\end{array}$} \\
\hline & & $\begin{array}{c}\text { Low } \\
\text { expressi } \\
\text { on }\end{array}$ & $\begin{array}{c}\text { High } \\
\text { expressi } \\
\text { on }\end{array}$ & & \\
\hline Sex & & & & 0.173 & 0.677 \\
\hline Male & 29 & 22 & 7 & & \\
\hline Female & 16 & 13 & 3 & & \\
\hline Age & & & & 0.649 & 0.421 \\
\hline$\leq 60$ & 18 & 14 & 4 & & \\
\hline$>60$ & 27 & 18 & 9 & & \\
\hline Tumor size & & & & 0.241 & 0.624 \\
\hline$\leq 2 \mathrm{~cm}$ & 15 & 12 & 3 & & \\
\hline$>2 \mathrm{~cm}$ & 30 & 22 & 8 & & \\
\hline Tumor site & & & & 0.741 & 0.390 \\
\hline Head of pancreas & 31 & 23 & 8 & & \\
\hline The body and tail of pancreas & 14 & 12 & 2 & & \\
\hline Differentiation grade & & & & 0.586 & 0.741 \\
\hline Low & 22 & 15 & 7 & & \\
\hline Moderate & 14 & 11 & 3 & & \\
\hline Hogh & 9 & 7 & 2 & & \\
\hline TNM stage & & & & 2.189 & 0.335 \\
\hline Stage I & 5 & 3 & 2 & & \\
\hline Stage II & 23 & 17 & 6 & & \\
\hline Stage III & 17 & 15 & 2 & & \\
\hline Distant metastasis & & & & 1.808 & 0.179 \\
\hline Yes & 21 & 15 & 6 & & \\
\hline No & 24 & 21 & 3 & & \\
\hline
\end{tabular}


Table 2 Cell lines used in experiments

\begin{tabular}{|c|c|c|c|}
\hline Cell line & Cell type & Resources & Country \\
\hline HPDE & $\begin{array}{c}\text { Pancreatic ductal } \\
\text { epithelium cell }\end{array}$ & $\begin{array}{c}\text { Cell bank of Chinese } \\
\text { Academy of Sciences }\end{array}$ & Shanghai, China \\
\hline PANC-1 & Pancreatic cancer cell & $\begin{array}{c}\text { Cell bank of Chinese } \\
\text { Academy of Sciences }\end{array}$ & Shanghai, China \\
\hline Bx-PC3 & Pancreatic cancer cell & $\begin{array}{c}\text { Cell bank of Chinese } \\
\text { Academy of Sciences }\end{array}$ & Shanghai, China \\
& & & \\
\hline
\end{tabular}

Table 3 Primer sequences used in the experiment

\begin{tabular}{|c|c|c|}
\hline $\begin{array}{l}\text { overexpres } \\
\text { sion }\end{array}$ & sense sequence & anti-sense sequence \\
\hline $\begin{array}{l}\text { miR-139-5p } \\
\text { mimic }\end{array}$ & $\begin{array}{c}\text { 5'-UCUACAGUGCACGUGUCUCCAG } \\
\text { U-3' }\end{array}$ & $\begin{array}{c}\text { 5'-UGGAGACACGUGCACUGUAGAU } \\
\text { U-3' }\end{array}$ \\
\hline SLC7A11 & $\begin{array}{c}\text { 5'-ATTTCATTCGAACGGAGATCTTG- } \\
3 \text {, }\end{array}$ & $\begin{array}{c}\text { 5'-CTAGCATGATCTCCGTTCGAATG- } \\
3 \text {, }\end{array}$ \\
\hline $\begin{array}{l}\text { luciferase } \\
\text { reporter }\end{array}$ & sense sequence & anti-sense sequence \\
\hline $\begin{array}{l}\text { SLC7A11- } \\
\text { UTR }\end{array}$ & $\begin{array}{c}\text { 5'-TGTTAGAAATAAGTATGGAGGAT } \\
-3^{\prime},\end{array}$ & $\begin{array}{l}\text { 5'-TATTATGTAAACTATCAAATGTTT } \\
\text { ATT-3' }\end{array}$ \\
\hline $\begin{array}{l}\text { SLC7A11- } \\
\text { UTR-mut }\end{array}$ & $\begin{array}{c}\text { 5'-GCCCATGAAAGACATTTTATTTA } \\
\text { AGTAGTATATATTCTTGCTTCACTT } \\
\text { TACC-3' }\end{array}$ & $\begin{array}{c}\text { 5'-GGTAAAGTGAAGCAAGAATATAT } \\
\text { ACTACTTAAATAAAATGTCTTTCAT } \\
\text { GGGC-3' }\end{array}$ \\
\hline qPCR & sense sequence & anti-sense sequence \\
\hline miR-139-5p & $\begin{array}{c}\text { 5'-GTCGTATCCAGTGCAGGGTCCGA } \\
\text { GG-3' }\end{array}$ & $\begin{array}{l}\text { 5'-TATTCGCACTGGATACGACCTGG } \\
\text { AG-3' }\end{array}$ \\
\hline SLC7A11 & 5'-GCTGTGATATCCCTGGCATT-3' & 5'-GGCGTCTTTAAAGTTCTGCG-3' \\
\hline U6 & 5'-CTCGCTTCGGCAGCACA-3' & 5'-AACGCTTCACGAATTTGCGT-3' \\
\hline GAPDH & $\begin{array}{c}\text { 5'-ACAACTTTGGTATCGTGGAAGG- } \\
3 \text {, }\end{array}$ & 5'-GCCATCACGCCACAGTTTC-3' \\
\hline
\end{tabular}


Table 4 Antibody information used in the experiment

\begin{tabular}{|c|c|c|c|}
\hline Antibody & WB & Specificity & Company \\
\hline GAPDH & $1: 1000$ & Rabbit monoclonal & Abcam, China \\
\hline SLC7A11 & $1: 1000$ & Rabbit Polyclonal & Abcam, China \\
\hline p-Akt & $1: 2000$ & Rabbit & CST, USA \\
\hline Akt & $1: 1000$ & Rabbit & CST, USA \\
\hline p-PI3K & $1: 1000$ & Rabbit & CST, USA \\
\hline PI3K & $1: 1000$ & Goat anti Rabbit & Abcam, China \\
\hline
\end{tabular}


A

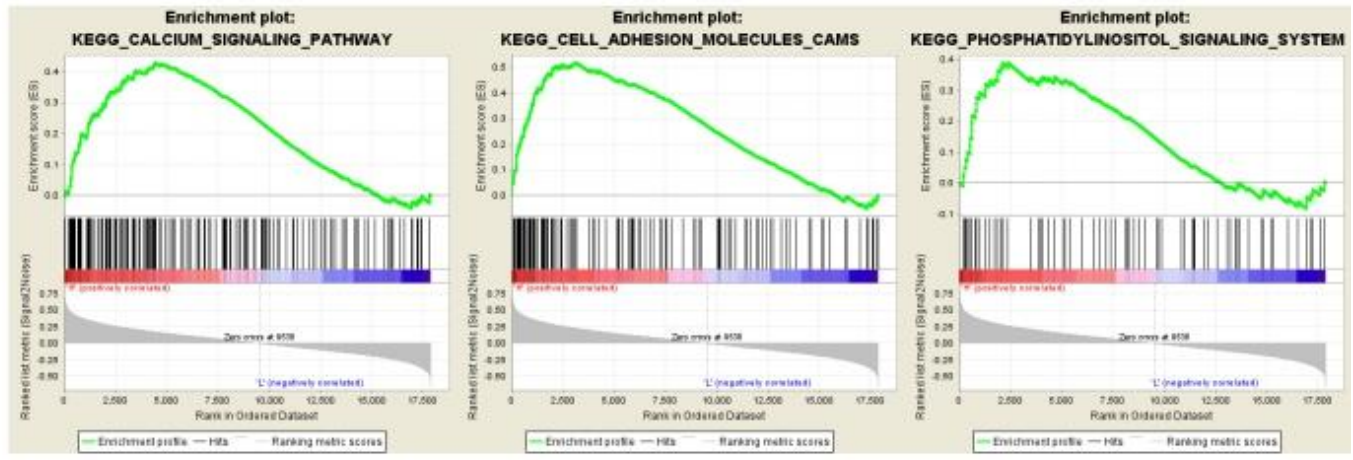

B

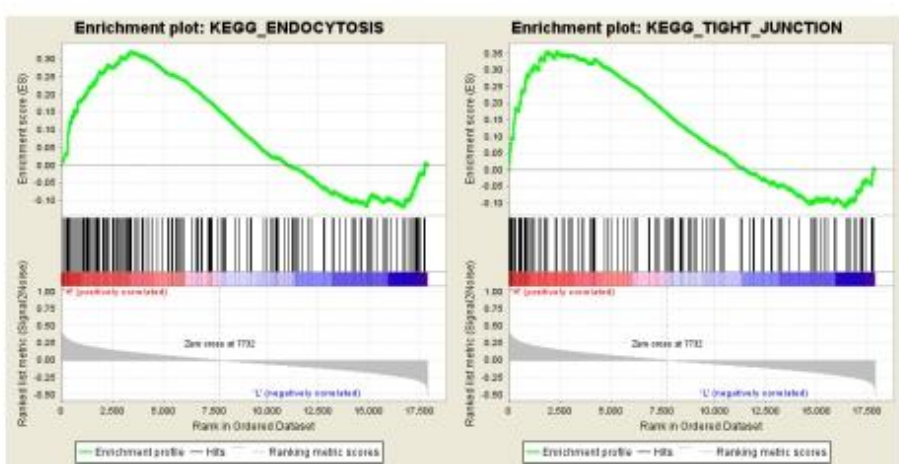

Attached map 1 Enrichment pathways of miR-139 and SLC7A11

A: GSEA pathway enrichment analysis results of miR-139; B: GSEA pathway enrichment analysis results of SLC7A11. 